\title{
Risk stratification for lymph node metastasis using Epstein-Barr virus status in submucosal invasive (pT1) gastric cancer without lymphovascular invasion: a multicenter observational study
}

\author{
Hiroki Osumi ${ }^{1} \cdot$ Hiroshi Kawachi ${ }^{2} \cdot K^{K a t s u y u k i ~ M u r a i}{ }^{3} \cdot$ Kimihide Kusafuka $^{4}$. Shuntaro Inoue ${ }^{5}$ Masaki Kitamura ${ }^{6}$ \\ Toshiyuki Yoshio ${ }^{1}$ (1) Naomi Kakusima ${ }^{3} \cdot$ Ryu Ishihara ${ }^{5} \cdot$ Hiroyuki Ono $^{3} \cdot$ Noriko Yamamoto $^{2} \cdot$ Takashi Sugino $^{4}$. \\ Shinichi Nakatsuka ${ }^{6} \cdot$ Satoshi Ida $^{7} \cdot$ Souya Nunobe ${ }^{7} \cdot$ Etsuro Bando $^{8} \cdot$ Takeshi Omori $^{9} \cdot$ Kengo Takeuchi $^{3}$. \\ Junko Fujisaki ${ }^{1}$
}

Received: 26 February 2019 / Accepted: 3 April 2019 / Published online: 6 May 2019

(c) The International Gastric Cancer Association and The Japanese Gastric Cancer Association 2019

\begin{abstract}
Background Lymphovascular invasion (LVI) is a strong predictive factor for lymph node metastasis (LNM) in early gastric cancer (GC). This study investigated the risk for LNM in pT1b GC without LVI based on Epstein-Barr virus (EBV) status in addition to conventional clinicopathological parameters.

Methods In total, 847 consecutive patients of pT1b GC without LVI who underwent surgery at three high-volume centers between 2005 and 2014 were retrospectively analyzed. Clinicopathological parameters and EBV status were evaluated, and univariate and multivariate analyses were performed to estimate LNM risk. With regard to the presence of those three parameters, risk stratification for LNM was performed and compared with a previously proposed risk classification that included low-risk ( $\mathrm{LNM}<3.0 \%$ ), intermediate-risk ( $\mathrm{LNM} \geq 3.0$ and $<19.6 \%$ ), and high-risk (LNM $\geq 19.6 \%)$ groups.

Results EBV-positive GC (EBVGC) accounted for 11.3\% (96 of 847) of cases; LNM was lower in EBVGC than in nonEBVGC (1 of $96,1.0 \%$ vs. 71/751, 9.5\%). In the multivariate analysis, non-EBVGC [odds ratio (OR) 10.8, 95\% confidence interval (CI) 1.48-78.9], age < 65 years (OR 2.13, 95\% CI 1.30-3.48), and tumor diameter $>3 \mathrm{~cm}$ (OR 2.26, 95\% CI 1.36-3.74) were independent risk factors for LNM. Patients with EBVGC were at low risk for LNM whereas those with all of three independent risk factors were at high risk (36 of 168, 21.4\%, 95\% CI 15.5-28.4).

Conclusion LNM risk stratification that includes EBV status is useful for clinical decision-making in pT1b GC cases without LVI.
\end{abstract}

Keywords Gastric cancer $\cdot$ pT1b $\cdot$ Lymphovascular invasion $\cdot$ Epstein-Barr virus $\cdot$ Lymph node metastasis

Electronic supplementary material The online version of this article (https://doi.org/10.1007/s10120-019-00963-7) contains supplementary material, which is available to authorized users.

Toshiyuki Yoshio

toshiyuki.yoshio@jfcr.or.jp

1 Department of Gastroenterology, The Cancer Institute Hospital of Japanese Foundation for Cancer Research, 3-8-31 Ariake, Koto-ku, Tokyo 135-8550, Japan

2 Department of Pathology, The Cancer Institute Hospital of Japanese Foundation for Cancer Research, Tokyo, Japan

3 Division of Endoscopy, Shizuoka Cancer Center, Shizuoka, Japan

4 Division of Pathology, Shizuoka Cancer Center, Shizuoka, Japan
5 Department of Gastrointestinal Oncology, Osaka International Cancer Institute, Osaka, Japan

6 Department of Pathology, Osaka International Cancer Institute, Osaka, Japan

7 Department of Gastroenterological Surgery, The Cancer Institute Hospital of Japanese Foundation for Cancer Research, Tokyo, Japan

8 Division of Gastric Surgery, Shizuoka Cancer Center, Shizuoka, Japan

9 Department of Gastroenterological Surgery, Osaka International Cancer Institute, Osaka, Japan 


\section{Background}

The progression in diagnosis and advancement of endoscopic therapy enable us to perform minimally invasive treatment, such as endoscopic submucosal dissection (ESD) [1]. The results of several retrospective multi-center studies [2-4] and prospective trials $[5,6]$ have led to the establishment of current treatment guidelines for early gastric cancer (EGC) [7]. The indication and curative resection criteria of ESD include several pathological factors such as histological type, tumor diameter, depth of invasion, accompanying ulceration, lymphovascular invasion (LVI), and resection margin [7].

Of those, there are robust data that LVI is the most important independent risk factor for lymph node metastasis (LNM) in EGC $[4,8]$ and is a non-curative factor for endoscopic therapy in the current treatment guidelines [7]. Meanwhile, in pT1b GC without LVI, the incidence of LNM is $9.8 \%[2,8]$ and surgical treatment is recommended even though $>90 \%$ of these cases do not have LNM. It is therefore important to develop more effective ways to stratify pT1b GC without LVI by analyzing previous known predictive factors for LNM and identifying new ones.

As a candidate factor, we focused on EBV status since its clinical implication has been previously reported [9-12] and EBV-positive GC (EBVGC) was recently defined as a distinct GC subtype by The Cancer Genome Atlas Research Network group based on the molecular profile [13]. We and others have shown that EBV status is a promising predictive factor for LNM in EGC $[12,14]$. Our previous study showed that the frequency of LNM was significantly lower in EBVGC than in non-EBVGC $(4.2 \%$ vs. $21.9 \%, P<0.0001)$ and in EBVGC, tumors without LVI exhibited a lower frequency of LNM than those with LVI ( 0 of $50,0 \%$; vs 3 of $21,14.3 \% ; P=0.023)[14]$. Furthermore, the incidence of LVI in EBVGC was very low compared to the non-EBVGC $[12,14]$. Therefore, EBVGC without LVI appears to be more suitable for further expansion of indication and curative resection criteria of ESD than EBVGC with LVI. However, due to the low rates of EBVGC, single-institution investigations with small study populations have not yielded definitive conclusions. A multi-institutional study with a larger cohort can overcome this limitation.

To this end, the present multicenter observational study aimed to establish an effective stratification method of LNM risk in pT1b GC without LVI by considering EBV status in addition to conventional parameters. We also examined the possibility of expanding the indication or curative resection criteria of ESD for pT1b GC.

\section{Materials and methods}

\section{Study population}

A total of 847 consecutive patients (576 men and 271 women) diagnosed with pT1b GC without LVI who underwent gastrectomy with regional lymph node dissection at The Cancer Institute Hospital of Japanese Foundation for Cancer Research, Shizuoka Cancer Center, and Osaka International Cancer Center between January 2005 and December 2014 were included in this study. LVI was determined based on hematoxylin and eosin (HE) stained tissue sections by expert pathologists in principal. To confirm the assessment, some specimens were subjected to D2-40 immunohistochemistry or Victoria blue-HE staining to detect lymphatic or venous invasion, respectively. Patients' clinical information was obtained from medical records. Exclusion criteria were as follows: (i) additional surgery after ESD; (ii) histological types other than adenocarcinoma-e.g. neuroendocrine carcinoma; (iii) multiple GCs including two or more with pT1b-T4; (iv) received neoadjuvant chemotherapy; (v) esophagogastric junction cancer; and (vi) insufficient clinicopathological information. This multi-institutional retrospective study was carried out in accordance with the Declaration of Helsinki and was approved by our institutional review board (registry no: 2017-1078). The study was conducted as part of the Knockout Submucosal Invasive GC (KOSMiC) Project, Japan.

\section{Tumor location, macroscopic types, and histopathological examination}

Tumor location was classified as upper-, middle-, and lower-third of the stomach according to the longitudinal position of the center of the tumor. Tumor type was macroscopically categorized according to the classification of the Japanese Gastric Cancer Association (JGCA) [15] as follows: 0-I, superficial elevated type; 0-IIa, superficial flat and elevated type; 0-IIb, superficial flat type; 0-IIc, superficial flat and depressed type; 0-III, superficial depressed type. When the tumor consisted of two or more of these components, the predominant type was used for classification. Histological type, depth of submucosal invasion, and complications of ulceration (including ulcer scar) were evaluated by experienced pathologists at each institute. For histological type, the JGCA classification [15] and two-tier classification proposed by Nakamura et al. [16]—which includes differentiated and undifferentiated types-were also used. The depth of submucosal invasion was measured from the bottom line of the muscularis mucosa to the 
invasive front according to the JGCA definition [7], and subsequently subclassified into pT1b1 (depth of submucosal invasion $<500 \mu \mathrm{m})$ and pT1b2 $(\geq 500 \mu \mathrm{m})$. When ulcerative changes or submucosal fibrosis with distorted muscularis mucosa were observed and not considered as being related to cancer invasion, they were regarded as complications of ulceration (ulcer or ulcer scars, respectively).

\section{Tissue microarray construction and in situ hybridization for EBV-encoded RNA (EBER-ISH)}

A single core was punched out of the representative tumor area (2-3 mm diameter) in each formalin-fixed, paraffinembedded tumor block and arrayed in a new tissue microarray block. Adjacent non-neoplastic tissue was included as an internal negative control. Sections with a thickness of $4 \mu \mathrm{m}$ were prepared from each block for hematoxylin and eosin staining and EBER-ISH, which was performed using a BOND ready-to-use ISH EBER probe and BOND III autostainer (Leica Biosystems Nussloch GmbH, Nussloch, Germany) according to the manufacturer's protocol.

\section{Statistical analysis}

Differences between pairs of groups were evaluated with the $\chi^{2}$ test, Fisher's exact probability test, or Mann-Whitney $U$ test. Uni- and multivariate logistic regression analyses were used to examine risk factors for LNM. In the multivariate analysis, all factors showing significance in the univariate analysis were simultaneously included in the model. Two-sided $P$ values $<0.05$ were considered statistically significant. Risk for LNM was stratified into lowrisk $(<3.0 \%)$, intermediate-risk $(\geq 3.0$ and $<19.6 \%)$, and high-risk ( $\geq 19.6 \%$ ) groups according to previous studies [17-19]. Statistical analyses were performed using EZR (Saitama Medical Center, Jichi Medical University) [20], a graphical user interface for R (The R Foundation for Statistical Computing, Vienna, Austria).

\section{Results}

\section{Relationship between clinicopathological parameters and LNM status}

The clinicopathological features of 847 pT1b GC cases according to LNM status are summarized in Table 1. Overall, 72 cases $(8.5 \%)$ showed LNM. There were no significant differences in LNM frequency among the three institutions $(P=0.478)$. Univariate analysis revealed that age less than 65 years, tumor diameter $>3 \mathrm{~cm}$, and nonEBVGC tumors were associated with LNM $(P=0.003$,
0.001 , and 0.002 , respectively). Moreover, all of these were independent risk factors for LNM in the multivariate analysis (Table 2).

Based on the three independent risk factors identified in the multivariate analysis (age, tumor diameter, and EBV status), we stratified our study cohort $(n=847)$ by combining these parameters. Patients with EBVGC were the lowrisk group for LNM [1/96, 1.0\%; 95\% confidence interval (CI): 0-5.7], whereas those with all three parameters constituted the high-risk group (36 of $168,21.4 \%$; $95 \%$ CI 15.5-28.4) and the remaining patients formed the intermediate-risk group (35 of 583, 6.0\%; 95\% CI 4.2-8.3).

\section{Clinicopathological and histological characteristics of pT1b EBVGC without LVI}

According to the EBER-ISH results, 96 of 847 pT1b GCs (11.3\%) were classified as being in the EBVGC. Comparisons of clinicopathological and histological parameters between the EBVGC and non-EBVGC revealed that EBVGC frequently occurred in men $(85.4 \%$ vs. $65.8 \%$; $P<0.0001)$ and was located in the upper-third region of the stomach $(47.9 \%$ vs. $19.1 \% ; P<0.0001)$ (Table 3 ). EBVGC also showed deeper submucosal invasion (median: 1000 vs. $750 \mu \mathrm{m} ; P=0.009$ ) and a lower rate of accompanying ulceration $(26.0 \%$ vs. $44.2 \% ; P=0.001)$. The frequency of LNM was lower in EBVGC than in non-EBVGC cases $(1.0 \%, 95 \%$ CI $0-5.7$ vs. $9.5 \%, 95 \%$ CI 7.5-11.8; $P=0.003$ ). Comparisons of histological type between EBVGC and non-EBVGC are shown in Supplemental Table 1. Although non-solid, poorly differentiated adenocarcinoma is the predominant histological type in both EBVGC and non-EBVGC according to the JGCA classification, its frequency was higher in EBVGC than in non-EBVGC cases (44 of 96, $45.8 \%$ vs. 233 of 751 , $31.0 \% ; P=0.004)$. Similarly, according to the Nakamura classification, the undifferentiated type was more frequent in EBVGC than in non-EBVGC cases (62 of 96, 64.6\% vs. 236 of $751,31.4 \% ; P<0.0001)$.

\section{Distribution of cases and relationship to clinicopathological factors}

The distribution of LNM rate, histological type, and submucosal invasion depth in each decade of patients' age are shown in Supplemental Table S2. Rates of LNM and undifferentiated histological type tended to decrease with age. Supplemental Table S3 shows the relationships among tumor diameter, histological type, and LNM. The rate of LNM tended to increase with tumor diameter in both differentiated and undifferentiated types. 
Table 1 Univariate analysis of clinicopathological and histological parameters for lymph node metastasis

\begin{tabular}{|c|c|c|c|c|}
\hline \multirow[t]{2}{*}{ Clinicopathological and histological paremeters } & \multirow[t]{2}{*}{ Total $(n=847)$} & \multicolumn{2}{|l|}{ Lymph node metastasis } & \multirow[t]{2}{*}{$P$ value } \\
\hline & & Positive $(n=72,8.5 \%)$ & Negative $(n=775,91.5 \%)$ & \\
\hline Median age, years (range) & $64(25-90)$ & $62(25-81)$ & $65(26-90)$ & 0.006 \\
\hline$<65$ & 434 & $49(11.3)$ & $385(88.7)$ & 0.003 \\
\hline$\geq 65$ & 413 & $23(5.6)$ & $390(94.4)$ & \\
\hline \multicolumn{5}{|l|}{ Sex, $n, \%$} \\
\hline Male & 576 & $45(7.8)$ & $531(92.2)$ & 0.294 \\
\hline Female & 271 & $27(10.0)$ & $244(90.0)$ & \\
\hline \multicolumn{5}{|l|}{ Location, $n, \%$} \\
\hline Upper third & 190 & $12(6.3)$ & $178(93.7)$ & 0.241 \\
\hline Middle or lower third & 657 & $60(9.1)$ & $597(90.9)$ & \\
\hline \multicolumn{5}{|l|}{ Macroscopic type (predominant), $n, \%$} \\
\hline $0-\mathrm{I}$ & 32 & $3(9.4)$ & $29(90.6)$ & 0.953 \\
\hline 0-IIa & 103 & $8(7.8)$ & $95(92.2)$ & \\
\hline 0 -IIb & 5 & $0(0)$ & $5(100)$ & \\
\hline 0-IIc & 707 & $61(8.6)$ & $646(91.4)$ & \\
\hline Median tumor diameter, mm (range) & $32(3-160)$ & $40(15-110)$ & $31(3-160)$ & 0.004 \\
\hline Diameter $\leq 3 \mathrm{~cm}$ & 398 & $21(5.3)$ & 377 (94.7) & 0.001 \\
\hline Diameter $>3 \mathrm{~cm}$ & 449 & $51(11.4)$ & $398(88.6)$ & \\
\hline \multicolumn{5}{|l|}{ Ulceration, $n, \%$} \\
\hline Absent & 490 & $36(7.3)$ & $454(92.7)$ & 0.171 \\
\hline Present & 357 & $36(10.1)$ & $321(89.9)$ & \\
\hline Median submucosal invasion depth, $\mu \mathrm{m}$ (range) & $750(10-13,000)$ & $900(50-6000)$ & $750(10-13,000)$ & 0.45 \\
\hline Depth $<500$ (pT1b1) & 293 & $18(6.1)$ & 275 (93.9) & 0.09 \\
\hline Depth $\geq 500$ (pT1b2) & 554 & $54(9.9)$ & $500(90.1)$ & \\
\hline \multicolumn{5}{|l|}{ Nakamura histological type (predominant), $n, \%$} \\
\hline Differentiated type & 411 & $29(7.1)$ & $382(92.9)$ & 0.175 \\
\hline Undifferentiated type & 436 & $43(9.9)$ & $393(90.1)$ & \\
\hline \multicolumn{5}{|l|}{ JGCA histological type (predominant), $n, \%$} \\
\hline pap & 14 & $1(7.1)$ & $13(92.9)$ & 0.582 \\
\hline tub1 & 179 & $11(6.1)$ & $168(93.9)$ & \\
\hline tub2 & 218 & $17(7.8)$ & $201(92.2)$ & \\
\hline por1 & 19 & $2(10.5)$ & $17(89.5)$ & \\
\hline por2 & 277 & $26(9.4)$ & $251(90.6)$ & \\
\hline sig & 135 & $14(10.4)$ & $121(89.6)$ & \\
\hline muc & 5 & $1(20.0)$ & $4(80.0)$ & \\
\hline \multicolumn{5}{|l|}{$\mathrm{EBV}, n, \%$} \\
\hline Negative & 751 & $71(9.5)$ & $680(90.5)$ & 0.002 \\
\hline Positive & 96 & $1(1.0)$ & $95(99.0)$ & \\
\hline
\end{tabular}

\section{Discussion}

In this study we incorporated EBV status into the risk stratification for LNM in pT1b GC without LVI, in addition to conventional risk factors. A multivariate analysis revealed that non-EBVGC, age $<65$ years, and tumor diameter $>3 \mathrm{~cm}$ were independent risk factors for LNM. We also confirmed that the combination of these parameters can stratify patients into three risk categories. Our results demonstrate that including EBV status into LNM risk stratification can improve the indication or curative resection criteria of EGC.

We found that EBV status is the strongest predictive factor for LNM risk among clinicopathological parameters in pT1b GC without LVI. Only a few studies have investigated the relationship between pT1b EBVGC without LVI and LNM in consecutive cases. Park et al. reported that among 592 pT1b GCs without LVI, 59 (10.0\%) were EBVGC and only one (1.7\%) showed LNM [12]. This current study evaluated a larger cohort (96 cases of pT1b EBVGC without LVI) and 
Table 2 Multivariate analysis of the three parameters for predicting risk of lymph node metastasis using a logistic regression model

\begin{tabular}{lll}
\hline Parameters & OR $(95 \%$ CI $)$ & $P$ value \\
\hline $\begin{array}{ll}\text { Median age, years (range) } \\
\geq 65\end{array}$ & 1 Ref & 0.0025 \\
$<65$ & $2.13(1.30-3.48)$ & \\
Tumor diameter, cm (range) & & \\
$\leq 3$ & 1 Ref & 0.0016 \\
$>3$ & $2.26(1.36-3.74)$ & \\
EBV & & \\
Positive & 1 Ref & 0.019 \\
Negative & $10.8(1.48-78.9)$ & \\
\hline
\end{tabular}

LNM frequency was also low (1 of 96, 1.0\%). Regarding lower frequency of EBVGC in GC (8.7-9.0\%) [11, 13], this multi-institutional study was able to obtain more reliable conclusion than previous reports although further validation study for much larger cohort can strengthen this.

In this study, age $<65$ years was an independent risk factor for LNM in the multivariate analysis. Clinicopathological differences in $\mathrm{GC}$ rates between young and elderly patients have been previously reported. GC in the elderly is mostly located in the lower third of the stomach and exhibits polyploidy or a superficial elevated gross histological type [21-24]. In contrast, infiltrating or superficial depressed gross histological type and poorer tumor differentiation were more common in younger patients [25-27]. Our data on tumor differentiation status were consistent with those in previous reports. However, to date there are no reports to indicate distinct differences of LNM frequency according to the age of patients with EGC. The significance of patient age in the present large cohort may determine the range of treatment options, especially for elderly patients with co-morbidities.

Tumor diameter is a predictive factor for LNM in EGC [7]. In this study, tumor diameter $>30 \mathrm{~mm}$ was an independent risk factor for LNM in the multivariate analysis. Tumor size $>30 \mathrm{~mm}$ and LVI were found to be correlated with increased risk of LNM in pT1b GC; these authors showed that lesions $\leq 30 \mathrm{~mm}$ in size, histologically differentiated type, lack of LVI, and submucosal invasion depth $<500 \mu \mathrm{m}$ (pT1b1) were completely free of LNM
Table 3 Comparison of clinicopathological and histological parameters between EBVGC and non-EBVGC cases in this study

\begin{tabular}{|c|c|c|c|}
\hline & EBVGC & Non-EBVGC & $P$ value \\
\hline Total number of cases & $96(11.3)$ & $751(88.7)$ & 0.61 \\
\hline Median age, years (range) & $63(46-88)$ & $64(25-90)$ & \\
\hline$<65$ & $53(55.2)$ & $381(50.7)$ & 0.448 \\
\hline$\geq 65$ & $43(44.8)$ & $370(49.3)$ & \\
\hline \multicolumn{4}{|l|}{ Sex, $n, \%$} \\
\hline Male & $82(85.4)$ & $494(65.8)$ & $<0.0001$ \\
\hline Female & $14(14.6)$ & $257(34.2)$ & \\
\hline \multicolumn{4}{|l|}{ Location, $n, \%$} \\
\hline Upper third & $46(47.9)$ & $144(19.1)$ & $<0.0001$ \\
\hline Middle or lower third & $50(52.1)$ & $607(80.9)$ & \\
\hline \multicolumn{4}{|l|}{ Macroscopic type, $n, \%$} \\
\hline 0 -I or 0 -IIa & $22(22.9)$ & $113(15.0)$ & 0.054 \\
\hline 0 -IIb or 0 -IIc & $74(77.1)$ & $638(85.0)$ & \\
\hline Median tumor diameter, mm (range) & $30(5-130)$ & $32(3-160)$ & 0.11 \\
\hline Diameter $\leq 3 \mathrm{~cm}$ & $53(55.2)$ & $345(45.9)$ & 0.1 \\
\hline Diameter $>3 \mathrm{~cm}$ & $43(44.8)$ & $406(54.1)$ & \\
\hline \multicolumn{4}{|l|}{ Ulceration, $n, \%$} \\
\hline Absent & $71(74.0)$ & $419(55.8)$ & 0.001 \\
\hline Present & $25(26.0)$ & $332(44.2)$ & \\
\hline Median submucosal invasion depth, $\mu \mathrm{m}$ (range) & $1000(50-13,000)$ & $750(10-8000)$ & 0.009 \\
\hline Depth $<500$ (pT1b1) & $23(24.0)$ & $271(36.1)$ & 0.022 \\
\hline Depth $\geq 500$ (pT1b2) & $73(76.0)$ & $480(63.9)$ & \\
\hline \multicolumn{4}{|l|}{ Lymph node metastasis, $n, \%$} \\
\hline Absent & $95(99.0)$ & $680(90.5)$ & 0.003 \\
\hline Present & $1(1.0)$ & $71(9.5)$ & \\
\hline
\end{tabular}

All cases in this study are pT1b gastric cancer without lymphovascular invasion $E B V G C$ Epstein-Barr virus-positive gastric cancer 
(95\% CI 0-2.5\%) [2]. Meanwhile, the incidence of LNM in undifferentiated type pT1b GCs was $23.8 \%$ (399 of 1680) and submucosal invasion depth $\geq 500 \mu \mathrm{m}$ (pT1b2) was an independent predictor of LNM by multivariate analysis [3]. However, no detailed evaluation of the relationship between tumor diameter and LNM incidence was performed. In this study, the LNM rate of pT1b GCs tended to increase with tumor diameter in both differentiated and undifferentiated histological types. Thus, in pT1b GC without LVI, tumor diameter is an important factor in LNM irrespective of the degree of differentiation.

We demonstrated that the stratification of GC patients based on three independent parameters including EBV status is useful for estimating the risk for LNM, which also aid decision-making in terms of the most suitable treatment strategy. In particular, EBVGC showed a lower LNM rate $(1 / 96,1.0 \%, 95 \%$ CI $0-5.7)$ corresponding to a lowrisk group. According to the current treatment guidelines, non-curative resection criteria after endoscopic resection include undifferentiated (poorly differentiated) histology, deeper submucosal invasion, presence of ulceration, larger tumor size, and presence of LVI [7]. The results of our study, however, indicate that the first three can be omitted in the risk assessment for LNM in cases of pT1b EBVGC without LVI. For instance, 94 of 96 (97.9\%) pT1b EBVGC cases in this study had at least one of the non-curative factors, and additional surgery would therefore be recommended under current treatment guidelines. Integrating EBV status and reconsidering the indication or curative resection criteria are expected to improve treatment outcome for EGC.

A limitation of this study is that the number of EBVGC cases was not large enough to achieve a conclusive guideline because the frequency of EBVGC was relatively low. Given that curative resection for $\mathrm{pT} 1 \mathrm{~b}$ GC tumors of expanded indication yielded an LNM rate with a $95 \%$ CI upper limit of $<3 \%$ in previous reports [2] and 5.7\% in EBV GC cases of this study, the results presented here must be verified in additional multi-institutional studies with a larger number of cases.

In conclusion, we demonstrated that including EBV status in LNM risk stratification in patients with pT1b GC without LVI is useful for establishing novel indication or curative resection criteria for EGC, which can inform clinical decision-making for personalized and more effective treatment.

Acknowledgments The authors thank Ms. Miyuki Kogure, Mr. Motoyoshi Iwakoshi, Ms. Tomoyo Kakita, Ms. Miki Hatta, Mr. Shuhei Ishii, and Ms. Naoko Takahashi for excellent technical support; and Dr. Maki Kobayashi for support and advice with the histological analysis.

Funding This research was supported by the Daiwa Securities Health Foundation, Takeda Japan Medical Office Funded Research Grant 2019, and a Japan Society for the Promotion of Science KAKENHI grant (no. JP16K08661).

\section{Compliance with Ethical Standards}

Conflict of Interest The authors declare that they have no conflict of interest.

Ethical approval All procedures performed in studies involving human participants were in accordance with the ethical standards of the institutional research committee (The Cancer Institute Hospital of Japanese Foundation for Cancer Research, Institutional Review Board; approval no. 2017-1078) and with the 1964 Helsinki declaration and its later amendments or comparable ethical standards.

Informed consent The protocol was described in the Web site of the hospital, and the subjects were provided with the opportunity to opt out, and therefore, no new consent was required from the patients.

\section{References}

1. Muto M, Yao K, Kaise M, Kato M, Uedo N, Yagi K, et al. Magnifying endoscopy simple diagnostic algorithm for early gastric cancer (MESDA-G). Dig Endosc. 2016;28:379-93.

2. Gotoda T, Yanagisawa A, Sasako M, Ono H, Nakanishi Y, Shimoda $\mathrm{T}$, et al. Incidence of lymph node metastasis from early gastric cancer: estimation with a large number of cases at two large centers. Gastric Cancer. 2000;3:219-25.

3. Hirasawa T, Gotoda T, Miyata S, Kato Y, Shimoda T, Taniguchi $\mathrm{H}$, et al. Incidence of lymph node metastasis and the feasibility of endoscopic resection for undifferentiated-type early gastric cancer. Gastric Cancer. 2009;12:148-52.

4. Hatta W, Gotoda T, Oyama T, Kawata N, Takahashi A, Yoshifuku $\mathrm{Y}$, et al. A scoring system to stratify curability after endoscopic submucosal dissection for early gastric cancer: "eCura system". Am J Gastroenterol. 2017;112:874-81.

5. Hasuike N, Ono H, Boku N, Mizusawa J, Takizawa K, Fukuda H, et al. A non-randomized confirmatory trial of an expanded indication for endoscopic submucosal dissection for intestinal-type gastric cancer (cT1a): the Japan Clinical Oncology Group study (JCOG0607). Gastric Cancer. 2018;21:114-23.

6. Takizawa K, Takashima A, Kimura A, Mizusawa J, Hasuike N, Ono $\mathrm{H}$, et al. A phase II clinical trial of endoscopic submucosal dissection for early gastric cancer of undifferentiated type: Japan Clinical Oncology Group study JCOG1009/1010. Jpn J Clin Oncol. 2013;43:87-91.

7. Japanese Gastric Cancer Association. Japanese gastric cancer treatment guidelines 2014 (ver. 4). Gastric Cancer 2017; 20: 1-19.

8. Sekiguchi M, Oda I, Taniguchi H, Suzuki H, Morita S, Fukagawa $\mathrm{T}$, et al. Risk stratification and predictive risk-scoring model for lymph node metastasis in early gastric cancer. J Gastroenterol. 2016;51:961-70.

9. Tokunaga M, Land CE. Epstein-Barr virus involvement in gastric cancer: biomarker for lymph node metastasis. Cancer Epidemiol Biomarkers Prev. 1998;7:449-50.

10. Fukayama M, Hino R, Uozaki H. Epstein-Barr virus and gastric carcinoma: virus-host interactions leading to carcinoma. Cancer Sci. 2008;99:1726-33.

11. Murphy G, Pfeiffer R, Camargo MC, Rabkin CS. Meta-analysis shows that prevalence of Epstein-Barr virus-positive gastric cancer differs based on sex and anatomic location. Gastroenterology. 2009;137:824-33.

12. Park JH, Kim EK, Kim YH, Kim JH, Bae YS, Lee YC, et al. Epstein-Barr virus positivity, not mismatch repair-deficiency, is a favorable risk factor for lymph node metastasis in submucosainvasive early gastric cancer. Gastric Cancer. 2016;19:1041-51. 
13. Cancer Genome Atlas Research Network. Comprehensive molecular characterization of gastric adenocarcinoma. Nature. 2014;513:202-9.

14. Osumi H, Kawachi H, Yoshio T, Ida S, Yamamoto N, Horiuchi Y, et al. Epstein-Barr virus status is a promising biomarker for endoscopic resection in early gastric cancer: proposal of a novel therapeutic strategy. J Gastroenterol. 2019. https://doi.org/10.1007/ s00535-019-01562-0.

15. Sano T, Aiko T. New Japanese classifications and treatment guidelines for gastric cancer: revision concepts and major revised points. Gastric Cancer. 2011;14:97-100.

16. Nakamura K, Sugano H, Takagi K. Carcinoma of the stomach in incipient phase: its histogenesis and histological appearances. Gan. 1968;59:251-8.

17. Sasako M, Kinoshita T, Maruyama K. Prognosis of early gastric cancer (in Japanese with English abstract). Stomach Intestine. 1993;28:139-46.

18. Sano T, Kobori O, Muto T. Lymph node metastasis from early gastric cancer: endoscopic resection of tumour. Br J Surg. 1992;79:241-4.

19. Ono H, Yao K, Fujishiro M, Oda I, Nimura S, Yahagi N, et al. Guidelines for endoscopic submucosal dissection and endoscopic mucosal resection for early gastric cancer. Dig Endosc. 2016;28:3-15.

20. Kanda Y. Investigation of the freely available easy-to-use software 'EZR' for medical statistics. Bone Marrow Transpl. 2013;48:452-8.
21. Habu H, Endo M. Gastric cancer in elderly patients-results of surgical treatment. Hepatogastroenterology. 1989;36:71-4.

22. Fujimoto S, Takahashi M, Ohkubo H, Mutou T, Kure M, Masaoka $\mathrm{H}$, et al. Comparative clinicopathologic features of early gastric cancer in young and older patients. Surgery. 1994;115:516-20.

23. Maehara Y, Emi Y, Tomisaki S, Oshiro T, Kakeji Y, Ichiyoshi Y, et al. Age-related characteristics of gastric carcinoma in young and elderly patients. Cancer. 1996;77:1774-800.

24. Wang JY, Hsieh JS, Huang CJ, Huang YS, Huang TJ. Clinicopathologic study of advanced gastric cancer without serosal invasion in young and old patients. J Surg Oncol. 1996;63:36-40.

25. Inoshita N, Yanagisawa A, Arai T, Kitagawa T, Hirokawa K, Kato Y. Pathological characteristics of gastric carcinomas in the very old. Jpn J Cancer Res. 1998;89:1087-92.

26. Arai T, Esaki Y, Inoshita N, Sawabe M, Kasahara I, Kuroiwa K, et al. Pathologic characteristics of gastric cancer in the elderly: a retrospective study of 994 surgical patients. Gastric Cancer. 2004;7:154-9.

27. Kitamura K, Yamaguchi T, Taniguchi H, Hagiwara A, Yamane $\mathrm{T}$, Sawai K, et al. Clinicopathological characteristics of gastric cancer in the elderly. Br J Cancer. 1996;73:798-802.

Publisher's Note Springer Nature remains neutral with regard to jurisdictional claims in published maps and institutional affiliations. 